\title{
Lymphocyte doubling time in chronic lymphocytic leukemia modern era: a real-life study in 848 unselected patients
}

Baumann, Tycho; Department of Hematology, Hospital Clinic, University of Barcelona, Spain Moia, Riccardo; Division of Hematology, Department of Translational Medicine, University of Eastern Piedmont, Novara, Italy

Gaidano, Gianluca; Division of Hematology, Department of Translational Medicine, University of Eastern Piedmont, Novara, Italy

Delgado, Julio; Department of Hematology, Hospital Clinic, University of Barcelona, Spain

Condoluci, Adalgisa; Division of Hematology, Institute of Oncology Research (IOR), Faculty of Biomedical Sciences, Università della Svizzera italiana, Switzerland

Villamor, Neus; Department of Pathology, Hospital Clinic, University of Barcelona, Spain

Payedimarri, Anil Babu; Division of Hematology, Department of Translational Medicine, University of Eastern Piedmont, Novara, Italy

Costa, Dolors; Cancer Cytogenetics, Department of Pathology, Hospital Clinic, Barcelona, Spain

Patriarca, Andrea; Division of Hematology, Department of Translational Medicine, University of Eastern Piedmont, Novara, Italy

Jiménez-Vicente, Carlos; Department of Hematology, Hospital Clinic, University of Barcelona, Spain

Rossi, Davide; Division of Hematology, Institute of Oncology Research (IOR), Faculty of Biomedical Sciences, Università della Svizzera italiana, Switzerland

Montserrat, Emili; Department of Hematology, Hospital Clinic, University of Barcelona, Spain

Leukemia, 4 Feb. 2020

Published version: https://doi.org/10.1038/s41375-021-01149-w

Postprint deposited in institutional repository RERO DOC

Università della Svizzera italiana - USI 
1 Title: Lymphocyte Doubling Time in Chronic Lymphocytic Leukemia Modern Era: A Real -Life

2 Study in 848 Unselected Patients

3 Running title: Lymphocyte Doubling Time in Chronic Lymphocytic Leukemia

4

5

6

7

8 (1) Department of Hematology. Hospital Clinic. University of Barcelona, Spain.

9 (2) Division of Hematology, Department of Translational Medicine, University of Eastern

10 Piedmont, 28100 Novara, Italy.

11 (3) Division of Hematology. Oncology Institute of Southern Switzerland.

12 (4) Department of Pathology. Hospital Clinic. University of Barcelona, Spain.

13 (5) Cancer Cytogenetics. Department of Pathology. Hospital Clinic. Barcelona, Spain

14 Tycho Baumann, current affilitation: Servicio de Hematología, Hospital 12 de Octubre, Avd de

15 Cordoba s/n., 28041 Madrid.

16

17

18

19

20

21

22

23

24

25

Correspondence:

Emili Montserrat, M.D.

Department of Hematology

Hospital Clínic, University of Barcelona

Villarroel, 170

08036 Barcelona, Spain

Tel. + 34932275475

E-mail: emontse@clinic.cat

The authors declare no conflict of interest.

T.B and R.M equally contributed to this manuscript
7

8 


\section{Abstract}

31 The prognostic significance of lymphocyte doubling time (LDT) in chronic lymphocytic

32 leukemia (CLL) was identified when the biology of the disease was poorly understood and

33 therapy was not effective. We assessed the clinical and biological significance of LDT in 848

34 CLL patients in a real-life setting and the context of new biomarkers and effective therapy. A

35 short LDT ( $\leq 12$ months) was enriched for adverse biomarkers. Patients with a rapid LDT did

36 need therapy shortly after diagnosis (median 23 months vs. not reached; $p<0.001$ ) and had a

37 poorer overall survival (median 95 months vs. not reached $p<0.001$ ). LDT, IGHV mutational

38 status, Beta-2 microglobulin, and Rai clinical stage were independent predictors for time to

39 first treatment in the whole series and in Binet stage A patients. No correlation was observed

40 between LDT and response to chemoimmununotherapy. However, a short LDT along with age

$41 \geq 65$ years, high-risk FISH (del(17p), del(11q)), unmutated IGHV, increased Beta-2

42 microglobulin, and TP53 mutations predicted short survival. Moreover, the prognostic

43 significance of LDT was independent of the CLL-IPI and the Barcelona/Brno prognostic model.

44 LDT remains an important outcome marker in the modern CLL era and should be incorporated

45 into the clinical assessment and stratification of CLL patients.

46 Abstract words: 200

47 Main text words: 2712

48 Number of references: 26

49 Key-words: Chronic lymphocytic leukemia, Lymphocyte doubling time, Tumor kinetics,

50 Biomarkers, Prognosis, Chemoimmunotherapy, Ibrutinib

51

52 
54 Tumor kinetics are an important determinant of the outcome of patients with cancer. In

55 chronic lymphocytic leukemia (CLL), the blood lymphocyte doubling time (LDT) reflects the

56 birth rate and pace at which neoplastic lymphocytes accumulate in the organism. LDT is a

57 validated independent biomarker that correlates with overall survival ${ }^{1-3}$. However, the seminal

58 studies on the significance of LDT in CLL were conducted when the understanding of the

59 biology of CLL was limited and treatment for this disease was not effective $e^{1,4-6}$.

60 The objectives of this study were twofold. First, to correlate LDT with new genetic and

61 molecular features of CLL; second, to determine the prognostic and predictive value of LDT in

62 the general population of CLL patients treated with effective regimens in daily practice.

64 Material and Methods

65 This is a retrospective observational study in 848 unselected CLL patients from two European

66 academic centers, the Hospital Clinic, University of Barcelona, Spain and the Amedeo Avogadro

67 University of Eastern Piedmont, Novara, Italy. Patients in all clinical stages (Binet A 780, Binet B

68 61; Binet C 6; Rai 0 614; Rai I+II 208; Rai III +IV 25) diagnosed between 2000 and 2016 were

69 included in the study. The study was approved by the local Institutional Review Boards and

70 was performed in accordance with the Declaration of Helsinki. LDT was measured the time

71 of diagnosis if prior WBC counts were available or calculated after diagnosis by linear

72 regression analysis, over a minimal observation, treatment-free period of 3 months ${ }^{1}$. Diagnosis

73 and criteria for starting therapy were as recommended by the International Workshop on CLL2.

74 Data at diagnosis retrieved from databases included age, sex, clinical stage, absolute

75 lymphocyte count, $\mathrm{Hb}$ level, platelet count, $\beta_{2}$-microglobulin (B2M) and lactate dehydrogenase

76 (LDH) levels. Fluorescent in situ hybridization (FISH) studies for del(11q), del(13q) and del(17p)

77 deletions and trisomy 12 were performed using the Vysis CLL probe kit (Abbott, Del Plains, IL, 
78 USA). IGHV rearrangements and mutational status were analyzed according to the European

79 Research Initiative on CLL (ERIC) recommendations ${ }^{7}$. Mutations of NOTCH1, SF3B1, ATM and

80 TP53 were determined using previously described methods ${ }^{8}$. Data were retrieved within the 3

81 months from diagnosis; in some cases, samples stored at diagnosis were retrospectively

82 analyzed. Due to the nature of the study, not all variables were available for all patients.

83 Although well balanced for most variables, patients from the Novara series were older (71 vs.

8467 years; $p<0.01$ ) and presented mutated IGHV more frequently than those from Barcelona

85 (70\% vs. 62\%; $p<0.02)$ (supplementary material Table 1 ). The median OS of the two series was

86 similar (144 months vs. 155 months $p>.05$ ). The main endpoint of the study was OS.

\section{Statistical methods}

88 Comparisons between groups were performed using the $\chi^{2}$ for continuous variables and Mann-

89 Whitney test for categorical variables. Overall survival (OS) was defined as the time between

90 diagnosis and the date of death or last follow-up using the Kaplan-Meier method. Time to first

91 treatment (TTFT) was calculated from the time of diagnosis to the time therapy was initiated

92 or patient's death. Survival curves were compared by the log-rank test. Analyses of the

93 independent prognostic value for OS and TTFT was performed with Cox regression multivariate

94 models. The minimal observation period before any event (initiation of treatment or death)

95 was 3 months. Actuarial plots were obtained after a landmark time of 12 months from

96 diagnosis and taking time of diagnosis as time 0 . Unadjusted $p$-values $<0.05$ were considered

97 statistically significant.

98

99

100

101

PAGE 13 


\section{Correlation of LDT with clinical features, biomarkers, and outcomes}

104 Among the 848 CLL patients, all clinical stages included, the proportion of patients with an 105 LDT $\leq 12$ months was 94 (11\%) and the proportion of patients with a LDT >12 months was 754

106 (89\%). The median follow-up up was 85 months (range 44-201) and 103.5 months (range 4-

107 224) for patients with a short and a long LDT, respectively. At the time of the analysis, 61/94

108 (65\%) patients with short LDT and 283/754 (38\%) of those with a long LDT had died.

109 The correlation of LDT ( $\leq 12$ months vs. > 12 months) with clinical features, biomarkers, and

110 outcomes is shown in Table 1. Patients with short LDT were predominantly male $(p=0.02)$, had

111 more advanced clinical stage $(p<0.001)$, higher absolute lymphocyte counts $(p<0.001)$ and

112 increased serum B2M $(p=0.004)$, and a tendency to increased serum LDH levels $(p=0.065)$.

113 Patients with short LDT had a trend towards lower levels of $\mathrm{Hb}$ and lower platelet counts, the

114 difference not being statistically significant. A short LDT was also associated with

115 unmutated IGHV status $(p<0.001)$ and poor FISH cytogenetics (del17p; $p=0.002$ and del11q;

$116 p<0.001)$. In addition, patients with short LDT presented more frequently mutations

117 in NOTCH1 ( $p=0.002)$, and TP53 $(p=0.012)$ as compared to those with a long LDT; similarly, a

118 tendency to a higher proportion of patients with SF3B1 $(p=0.064)$ and ATM mutations

$119(p=0.102)$ was observed in patients with short LDT.

120 In contrast, patients with a long LDT were enriched for initial clinical stages (Binet A, Rai 0),

121 del(13q), lower blood lymphocyte counts and normal FISH analysis (Table1). LDT did not

122 predict response to initial therapy, which in $50 \%$ of cases consisted of chemoimmunotherapy

123 (FCR, BR, Chlorambucil + anti-CD20 mononoclonal antibodies) (ORR 73\% (CR 44\%) vs. ORR 66\%

124 (CR 36\%); $p=0.326$ ) (Table 1 and supplementary material Table 2). 


\section{LDT and time-to-first therapy}

128 Altogether, 310 of 848 patients (37\%) required therapy. All patients considered, the median

129 TTFT was 174 months. Patients with a short LDT needed therapy more frequently (77/94 or

$13082 \%$ vs. $233 / 754$ or $31 \%)(p<0.001$ ) and more rapidly (median TTFT 23 months (range 16-30)

131 vs. not reached) $(p<0.001)$ than those with a long LDT, independently of clinical stage (Figure

$1321 \mathrm{~A}$ and supplementary material Table 3). Among 780 patients with Binet stage $A$ disease, the

133 median TTFT for those with a short LDT ( $n=76)$ was 25 months (17-32) while in patients with a

134 long LDT $(n=704)$ the median TTFT had not been reached $(p<0.001)$, independently of Rai

135 stage. In 61 patients with Binet stage B disease, the median TTFT in cases with a short LDT

$136(n=17)$ was 12 months (2-22) vs. 34 months (20-48) in those with a long LDT $(n=44)(p=0.074)$,

137 independently of Binet clinical stage. In 61 patients with Binet stage B disease, the median

138 TTFT in cases with a short LDT ( $n=17)$ was 12 months (range 2-22) vs. 34 months (range 20-48)

139 in those with a long LDT $(n=44)(p=0.074)$. There were only 6 patients in Binet stage $C$ disease,

140 precluding a meaningful analysis of this group of patients (supplemental material Table 3).

141 Results did not significantly differ when considering Rai instead of Binet clinical stages (data

142 not shown). In addition, no differences were observed when the actuarial plots were

143 obtained from the time of diagnosis or after a landmark of 12 months after diagnosis

144 (supplementary material Figure 2A).

145 In the univariate analysis, biomarkers correlated with a shorter time to first treatment (TTFT)

146 were advanced Rai clinical stage, increased B2M, short LDT, unmutated IGHV, high risk FISH 
148 cytogentics as defined by presence of del(11q) or del(I17)p, mutations in NOTCH1, SF3B1, and

149 ATM (all $\mathrm{p}<0.001)$, as well as mutations in TP53 ( $p=0.003)$ (Table 2 and supplementary Table 150 5).

151 In multivariate analysis including age, Rai stage, B2M, LDT, IGHV, high-risk FISH cytogenetics,

152 and mutations in TP53, NOTCH1, SF3B1, and ATM, a short LDT maintained its independent

153 prognostic value for TTFT (HR 4.3 (95\% Cl: 3.0-6.1), p<0.001) along with Rai stage (HR 2.6 (95\%

$154 \mathrm{Cl}: 2.0-3.5), p<0.001)$, B2M (HR 1.5 (95\% Cl: 1.1-2.1), $p=0.005)$, IGHV mutations (HR 3.0 (95\%

$155 \mathrm{Cl}: 2.3-4.1), p<0.001)$, NOTCH1 mutations (HR 1.6 (95\% Cl: 1.1-2.4), $p=0.012$ ), and SF3B1

156 mutations (HR 1.99 (95\% Cl: 1.2-2.9), $p=0.008$ ) (Table 2 and supplementary Table 5).

\section{LDT and overall survival}

159 At the time of the analysis, 359 patients had died. The median follow-up of surviving patients

160 was 100 months (range 4-224). The median OS of the whole series was 150 months (range 5-

161 224). In those subjects with a LDT $\leq 12$ months ( $n=94)$, the median OS was 95 months (range:

162 15-201) in comparison to 161 months (range: 5-224) in patients with a LDT > 12 months

$163(n=754)(p<001)$ (Figure 1), independently of clinical stages (supplementary material Table 4).

164 In an exploratory analysis LDT showed a tendency to behave as a continuous variable

165 (supplementary material Figure 1).

166 To investigate the prognostic significance of LDT uni- and multivariate adapted analyses were

167 performed (Table 2 and supplementary material Table 6). In univariate analysis there was a

168 correlation between OS and advanced clinical stage (Rai 0 vs. I-IV), age $\geq 65$ years, increased

169 B2M, higher LDH, short LDT, unmutated IGHV, and high-risk FISH genetics (del(17p) and/or

170 del(11q)) (all $p<0.001)$. Likewise, mutations in NOTCH1 $(p<0.001)$ and TP53 $(p<0.001)$ were

171 associated with OS while no association was found for SF3B1 $(p=0.25)$ and ATM $(p=0.35)$. 
172 In multivariate analysis including age, Rai stage, B2M, LDT, IGHV, FISH cytogenetics (alterations 173 of del(17p) or del(11q)), TP53 mutations, and NOTCH1 mutations, a short LDT maintained its 174 prognostic value for OS (HR $1.5(95 \% \mathrm{Cl}: 1.1-2.1), p<0.017)$ along with age $\geq 65$ years (HR 2.9 175 (95\% Cl: 2.1-3.9), p<0.001), high-risk FISH (del(17p), del(11q))(HR 1.9 (95\% Cl: 1.31762.8 ), $p=0.002$ ), unmutated IGHV (HR 1.8 (95\% Cl: 1.3-2.3), p<0.001), increased B2M (HR 2.1 177 (95\% Cl: 1.5-2.8), $p<0.001$ ), and TP53 mutations (HR 1.6 (95\% Cl: 1.1-2.6), $p=0.045$ ) (Table 2).

178 Next, we investigated whether LDT added prognostic value to CLL-IPI or to the Barcelona/Brno 179 prognostic model ${ }^{9,10}$. For this, we first performed multivariate analysis including LDT and CLL180 IPI variables (age, B2M, IGHV, del(17p) or TP53 alteration, Rai stage). LDT maintained its 181 prognostic value for OS (HR $1.6(95 \% \mathrm{Cl} 1.2-2.3), \mathrm{p}=0.003)$ along with age $\geq 65$ years (HR 3.0 182 (95\% Cl: 2.2-4.0), $p<0.001)$, increased B2M (HR 2.4 (95\% Cl: 1.9-3.2), $p<0.001$ ), unmutated $183 \operatorname{IGHV}(\mathrm{HR} 1.9(95 \% \mathrm{Cl}: 1.5-2.5), p<0.001)$, and presence of alterations in 17p and/or TP53 (HR 1841.9 (95\% Cl: 1.3-2.8), $p=0.001$ ) while Rai stage failed to enter the OS prognostic model. 185 ( $p>0.05)$ (supplementary material Table 6). Additionally, in a multivariate analysis including 186 LDT together with Barcelona-Brno variables (IGHV mutational status and FISH analysis). LDT 187 showed independent prognostic value for OS (HR 1.6 (95\% Cl: 1.2-2.2), $p=0.002$ ) along with 188 unmutated IGHV (HR 1.9 (95\% Cl: 1.5-2.4), $p<0.001)$ and high-risk FISH cytogenetics 189 (del(17p)/del(11q)) (HR 1.8 (95\% Cl: 1.3-2.5), $p<0.001)$ (supplementary material Table 6). As for 190 OS, no differences were observed when the actuarial plots were obtained from the time of 191 diagnosis or after a landmark of 12 months (supplementary material Figure 2B).

192 193 194 195 
196

PAGE 13 
198 CLL is characterized by a heterogeneous clinical course and variable response to therapy. Due

199 to this, the management of patients with CLL highly relies on prognostic factors (which

200 estimate overall survival) and predictive factors (which anticipate response to a given

201 treatment). Age, clinical stage (i.e., Rai or Binet), serum B2M, IGHV mutational status,

202 cytogenetic features (e.g., del(17p)/TP53 mutations, del(11q)) are considered the most

203 relevant outcome biomarkers ${ }^{11,12}$

204 In the last decade, progress in the understanding of the biology and therapy of CLL has led to

205 the identification of a huge number of potential biomarkers, although most of them have not

206 been incorporated into daily clinical practice due their complexity, limited availability, lack of

207 validation, or arguable clinical usefulness. In this regard, it is important to underline that

208 biomarkers should be easily obtained, reproducible, and biologically and clinically meaningfu $\left.\right|^{12}$

209 In 1966, in a seminal study, David Galton scrutinized a large series of CLL patients with a long

210 follow-up and observed several "blood lymphocyte trends" (from stable to rapidly increasing)

211 which correlated with the clinical course of CLL, from a benign to an aggressive disorder ${ }^{13}$. This

212 observation was at the origin of the identification of LDT as a biomarker in $\mathrm{CLL}^{1-6}$. LDT is easily

213 calculated and applied, is reproducible, and can be used in any setting. Moreover, LDT is a

214 biologically meaningful biomarker as it is mechanistically related to the replication and

215 expansion of neoplastic lymphocytes ${ }^{14,15}$, CLL cells birth-rate ${ }^{16,17}$, driver mutations ${ }^{18}$, and

216 genomic aberrations ${ }^{18}$.

217 There is a renewed interest on LDT in the context of progress in CLL molecular biology and

218 therapy ${ }^{19,20}$. The German CLL Study Group (GCLLSG) has published a study based on 539 stage

219 A patients from the CLL1 trial ${ }^{21}$. In this study, LDT emerged as a significant, independent

220 prognostic biomarker for TTFT and OS. Other independent prognostic parameters were

221 del(17p), unmutated IGHV, B2M $>3.5 \mathrm{mg} / \mathrm{dL}$, and age $>60$ years, which are the building block 
222 for the $\mathrm{CLL}^{-I P I}{ }^{9}$; del(11q) was also found to be prognostically significant; these results are

223 validated in our study. Based on their results, the GCLLSG elaborated a prognostic index that

224 efficiently discriminates four prognostic groups.

225 Our study is the first comprehensive analysis of LDT in the modern CLL era. We found that LDT

226 captures a wide array of adverse (short LDT) or favorable (long LDT) biomarkers, which

227 explains its robustness as biomarker. Also, LDT is an independent biomarker for TTFT and OS.

228 The prognostic significance of LDT is relevant because of its simplicity and independence from

229 other biomarkers and CLL outcome models $s^{9,10}$, and thus can be used in conjunction with

230 them. Whether LDT could be employed as a surrogate for IGHV mutational status has been

231 raised ${ }^{22}$. This is not supported by our data since $35 \%$ of our patients with a short LDT had

232 mutated IGHV. Likewise, the proportion of patients with no TP53 aberrations and a short LDT

233 was 35\%. Thus, LDT may complement but not replace IGHV mutational status nor TP53

234 aberrations in CLL prognostication.

235 In our study, LDT did not correlate with response to chemoimmunotherapy, which agrees with

236 a recent report in patients treated with $\mathrm{FCR}^{23}$. This strongly suggests that differences in OS

237 according to LDT are due to the heterogeneous biology of the disease, which is revealed by

238 LDT, rather than by differences in treatment response rates.

239 Historically, the usefulness of LDT as biomarker has been questioned on the basis that in many

240 cases it is not available at the time of diagnosis and that a short LDT is a criterion to initiate

241 therapy. However, CLL rarely constitutes a treatment emergency. Indeed, it is recommended

242 that after diagnosis patients are observed for 4-8 weeks to complete the diagnostic workup

243 and to assess the pace of the disease, this including LDT if not available. On the other hand, a

244 short LDT is infrequently the only reason to start therapy ( $<1 \%$ of cases in the Barcelona series

$\left.245{ }^{24}\right)$. In this regard, it is also worth emphasizing that in our analysis either time of diagnosis or a 
24612 months landmark after diagnosis were used as time 0 in statistical analysis, with no

247 significant differences.

248 This paper has the limitations inherent to all retrospective analysis, including that not all

249 biomarkers were available in the entire cohort of patients. Also, the proportion of patients

250 treated upfront with BTK and BCL2 inhibitors was quite small. In a limited independent cohort

251 of patients initially treated with ibrutinib, a raw analysis showed a likely correlation between

252 LDT and OS (supplementary material Figure 3). However, the prognostic and predictive value

253 of LDT in patients treated with ibrutinib must be prospectively determined in large series of

254 patients. The most important strength of this paper is the demonstration that LDT remains

255 an important marker for OS in the modern CLL era.

256 The fact that the conclusions from this paper are based on OS rather than progression-free-

257 survival (PFS) is important and deserves comment. Thus, in most cases CLL runs a protracted

258 clinical course characterized by consecutive episodes of disease progression and need for

259 therapy. Consequently, the OS depends on the response to different treatments given during

260 the disease. Therefore, progression-free-survival (PFS), which largely depends on treatment

261 modality, cannot replace OS as ultimate endpoint ${ }^{25,26}$. Nevertheless the relationship of LDT

262 and PFS in cohorts of homogeneously treated patients within trials warrants study.

263 In conclusion, this study shows that LDT (1) significantly correlates with CLL biomarkers, a

264 short and a long LDT being significantly enriched for adverse and favorable biomarkers,

265 respectively; (2) remains an independent biomarker for TTFT and OS in the modern CLL era;

266 and (3) pending of further study, it does not appear to be a treatment-dependent biomarker.

267 For these reasons and because of its applicability in all settings, it is advisable to include LDT in

268 the assessment and stratification of CLL patients and in prognostic studies pursuing the

269 identification of new biomarkers for this form of leukemia. 
271

272

273

274

275

276

277

278

279

280

281

282

283

284

285

PAGE 13 


\section{Acknowledgments}

287 This study was supported by Janssen research grant; Swiss Cancer League, ID 3746, 4395 4660,

288 and 4705, Bern, Switzerland; European Research Council (ERC) Consolidator Grant CLLCLONE,

289 ID: 772051; Swiss National Science Foundation, ID 320030_169670/1 and 310030_192439,

290 Berne, Switzerland; The Leukemia \& Lymphoma Society, Translational Research Program, ID

291 6594-20, New York.

292 AIRC 5 × 1000 No. 21198, Associazione Italiana per la Ricerca sul Cancro Foundation Milan,

293 Italy; the AGING Project - Department of Excellence - DIMET, Università del Piemonte

294 Orientale, Novara, Italy; Ricerca Finalizzata 2018 (project RF-2018-12365790), MoH, Rome,

295 Italy

296

297

298 The authors declare no conflict of interest.

299

300

301

302

303

304

305

306 
309 1.Montserrat E, Sánchez-Bisono J, Viñolas N. Rozman C. Lymphocyte doubling time in chronic

310 lymphocytic leukaemia: analysis of its prognostic significance. British Journal of Haematology 311 1986; 62: 567-575.

312 2.Hallek M, Cheson BD, Catovsky D, Caligaris-Cappio F, Dighiero G, Döhner H et al. iwCLL

313 guidelines for diagnosis, indications for treatment, response assessment, and supportive

314 management of CLL. Blood 2018; 131:2745-2760. doi:10.1182/blood-2017-09-806398

315 3.Eichhhorst B, Robak T, Montserrat E, Ghia P, Niemann C, Kater AP et al. on behalf of the

316 ESMO Guidelines Committee. Chronic lymphocytic leukaemia: ESMO Clinical Practice

317 Guidelines for diagnosis, treatment and follow-up. Ann Oncol 2020 in press.

318 4.Molica S, Alberti A. Prognostic value of the lymphocyte doubling time in chronic

319 lymphocytic leukemia. Cancer 1987; 60;2 712-2716.

320 5.Dhodapkar M, Tefferi A, Su J, Phyliky RL. Prognostic features and survival in young adults

321 with early/intermediate chronic lymphocytic leukemia (B-CLL): a single institution

322 study. Leukemia 1993;7(8):1232-1235.

323 6.Axdorph U, Nilsson BI, Nilsson BR, Björkholm M. X Leucocyte doubling time is a useful

324 predictor of progression-free survival in chronic lymphocytic leukaemia. Journal of Internal

325 Medicine, 1995; 237: 205-209. doi:10.1111/j.1365-2796.1995.tb01162.x

326 7.Ghia P, Stamatopoulos K, Belessi C, Moreno C, Stilgenbauer S, Stevenson F et al. ERIC

327 recommendations on IGHV gene mutational status analysis in chronic lymphocytic

328 leukemia. Leukemia. 2007;21(1):1-3. doi:10.1038/sj.leu.2404457

329 8. Puente XS, Pinyol M, Quesada V, Conde L, Ordóñez GR, Villamor N et al. Whole- genome 330 sequencing identifies recurrent mutations in chronic lymphocytic leukaemia. Nature 331 2011;475:101-105. doi:10.1038/nature10113. 
332 9.International CLL-IPI working group. An international prognostic index for patients with

333 chronic lymphocytic leukaemia (CLL-IPI): a meta-analysis of individual patient data. Lancet

334 Oncol. 2016;17:779-790. doi:10.1016/S1470-2045(16)30029-8.

335 10.Delgado J., Doubek M., Baumann T., Kotaskova J, Molica S, Mozas P et al. Chronic

336 lymphocytic leukemia: A prognostic model comprising only two biomarkers (IGHV mutational

337 status and FISH cytogenetics) separates patients with different outcome and simplifies the CLL-

338 IPI. American Journal of Hematology. 2017;92:375-380. doi: 10.1002/ajh.24660.

339 11.Rossi D, Gerber B, Stüssi G. Predictive and prognostic biomarkers in the era of new targeted

340 therapies for chronic lymphocytic leukemia. Leuk Lymphoma. 2017;58(7):1548-1560.

341 doi:10.1080/10428194.2016.1250264

342 12.Montserrat E, Gale RP. Predicting the outcome of patients with chronic lymphocytic

343 leukemia: Progress and uncertainty. Cancer 2019; 125:3699-3705. doi:10.1002/cncr.32353

344 13. Galton DAG. The pathogenesis of chronic lymphocytic leukemia. Canad Med Ass J 1966; 94:

$345 \quad 1005-1010$

346 14.Theml H, Ziegler-Heitbrock HWL. Management of CLL and allied disorders with reference to 347 their immunology and proliferation kinetics. Recent Results in Cancer Research 1984; 93: 240348258

349 15.Simonsson B, Nilsson K. 3H-Thymidine uptake in chronic lymphocytic leukemia cells. Scand J 350 Hematol 1980: 24: 169-173.

351 16.Orfao A, Ciudad J, González M, San Miguel M, García AR, López-Berges MC et al. Prognostic 352 value of S-phase white blood cell count in B-cell chronic lymphocytic leukemia. Leukemia 1992; $3536: 47-51$ 
354 17.Messmer BT, Messmer D, Allen SL, Kolitz JE, Kudalkar P, Cesar D et al. In vivo measurements

355 document the dynamic cellular kinetics of chronic lymphocytic leukemia B cells. J Clin Invest.

356 2005;115(3):755-764. doi:10.1172/JCI23409

357 18.Gruber M, Bozic I, Leschiner I, Livitz D, Stevenson K, Rassenti L et al. Growth dynamics in

358 naturally progressing chronic lymphocytic leukaemia. Nature 2019; 570: 474-479.

359 doi:10.1038/s41586-019-1252-x

360 19.Nadeu F, Diaz-Navarro A, Delgado J, Puente XS, Campo E. Genomic and Epigenomic

361 Alterations in Chronic Lymphocytic Leukemia. Annu Rev Pathol. 2020;15:149-177.

362

doi:10.1146/annurev-pathmechdis-012419-032810

363

364

365

20.Burger J. Treatment of chronic lymphocytic leukemia New England Journal of Medicine 2020;383:460-73. DOI: 10.1056/NEJMra1908213

21. Hoechstetter MA, Busch R, Eichhorst B, Bühler A, Winkler D, Bahlo J et al. Prognostic model for newly diagnosed CLL patients in Binet stage A: results of the multicenter, prospective CLL1 trial of the German CLL study group. Leukemia. 2020. 34(4):1038-1051. doi:10.1038/s41375-020-07272

22. Lad DP, Tejaswi V, Jindal N, Malhotra P, Khadwal A, Prakash G et al. Modified CLL International Prognostic Index (CLL-LIPI) using lymphocyte doubling time (LDT) in place of IgHV mutation status in resource limited settings predicts time to first treatment and overall survival, Leukemia \& Lymphoma 2020, 61: 1512-1515, DOI: 10.1080/10428194.2020.1719099 https://doi.org/10.1080/10428194.2020.1719099

23. Herling CD, Cymbalista F, Groß-Ophoff-Müller C, Bahlo J, Robrecht S, Langerbeins P et al. Early treatment with FCR versus watch and wait in patients with stage Binet A high-risk chronic lymphocytic leukemia (CLL): a randomized phase 3 trial [published online ahead of print, 2020 Feb 18]. Leukemia. 2020;10.1038/s41375-020-0747-7. doi:10.1038/s41375-020-0747-7 
378 24. Mozas P, Rivas-Delgado A, Baumann T, Villamor N, Ortiz-Maldonado V, Aymerich M et al.

379 Analysis of criteria for treatment initiation in chronic lymphocytic leukemia. Cancer Journal

$380 \quad 2018 ; 8: 1-5$

381 25. Prasad V, Kim C, Burotto M, Vandross A. The strength of association between surrogate

382 end points and survival in oncology: A systematic review of trial-level meta-analyses. JAMA

383 Intern Med. 2015;175(8):1389-1398. doi:10.1001/jamainternmed.2015.2829 19.

384 26. Eichhorst, B, Fink AM, Bahlo J, Busch R, Kovacs G, Maurer Ch et al. First-line

385 chemoimmunotherapy with bendamustine and rituximab versus fludarabine,

386 cyclophosphamide, and rituximab in patients with advanced chronic lymphocytic leukaemia

387 (CLL10): international, open-label, randomised, phase 3, non-inferiority trial. Lancet Oncol

388 2016; 17: 928-942. Published Online May 20, 2016

389

390

391

392

393

394

395

396

397

398

399

PAGE 13 
400

401 Figure legends

402

403 Figure 1A. Time-to-first therapy (TTFT) of patients with CLL according to LDT (median 23

404 months vs. not reached; $p<0.001)$.

405

406 Figure 1B. Overall survival (OS) of patients with CLL according to LDT (median 95 months vs.

407161 months; $p<0.001) .1919$

$408 \quad 19$ 
Table 1. Demographics, clinico-biological features, and outcomes in 848 patients according to LDT

\begin{tabular}{|c|c|c|c|}
\hline & $\begin{array}{c}\text { LDT } \leq 12 \text { months } \\
n=94\end{array}$ & $\begin{array}{c}\text { LDT >12 months } \\
\quad n=754\end{array}$ & $P$ \\
\hline \multicolumn{4}{|l|}{ Age } \\
\hline Median (range) & $69(40-90)$ & $69(34-100)$ & .93 \\
\hline$\leq 65$ years $(\%)$ & $42 / 94(45)$ & $301 / 754(40)$ & .375 \\
\hline Gender, male (\%) & $64 / 94(68)$ & $418 / 754(55)$ & .02 \\
\hline Rai, 0 vs I-IV (\%) & 42 vs 156 ( 45 vs 55$)$ & 572 vs 181 (76 vs 24$)$ & $<.001$ \\
\hline Lymphocyte count, $\times 10^{9} / \mathrm{L}$ (mean \pm SD) & $20.7 \pm 23.2$ & $11.7 \pm 11.6$ & $<<.001$ \\
\hline $\mathrm{Hb}, \times 10^{9} / \mathrm{L}($ mean $\pm \mathrm{SD})$ & $13.7 \pm 1.3$ & $13.9 \pm 1.5$ & .662 \\
\hline Platelets, $\times 10^{9} / \mathrm{L}($ mean \pm SD) & $205 \pm 69$ & $217 \pm 72$ & .109 \\
\hline LDH increased (\%) & $15 / 94(16)$ & $69 / 746(9)$ & .065 \\
\hline B2M increased (\%) & $57 / 91(63)$ & $337 / 727(46)$ & .004 \\
\hline Unmutated IGHV (\%) & $53 / 82(65)$ & $192 / 637(30)$ & $<.001$ \\
\hline \multicolumn{4}{|l|}{ FISH } \\
\hline del13q (\%) & $48 / 92(52)$ & $366 / 696(53)$ & 1.0 \\
\hline Normal (\%) & $15 / 93(16)$ & $228 / 698(33)$ & .001 \\
\hline Tris12 (\%) & $23 / 93(25)$ & $117 / 687(17)$ & .083 \\
\hline del11q (\%) & $17 / 92(19)$ & $40 / 694(6)$ & $<.001$ \\
\hline del17p (\%) & $11 / 92(12)$ & $26 / 694(4)$ & .002 \\
\hline Complex karyotype (\%) & $3 / 42(7)$ & $23 / 337(7)$ & 1.0 \\
\hline NOTCH1 mutated (\%) & $16 / 73(22)$ & $47 / 540(9)$ & .002 \\
\hline SF3B1 mutated (\%) & $8 / 70(11)$ & $29 / 526(6)$ & .064 \\
\hline ATM mutated (\%) & $7 / 65(11)$ & $29 / 524(6)$ & .102 \\
\hline TP53 mutated (\%) & $11 / 75(15)$ & $34 / 576(6)$ & .012 \\
\hline Treatment (\%) & $77 / 94$ (82) & $233 / 754$ (31) & $<.001$ \\
\hline
\end{tabular}




\begin{tabular}{|c|c|c|c|}
\hline Chemoimmunotherapy (\%) & $38 / 77(49)$ & $117 / 233(50)$ & 1.0 \\
\hline \multicolumn{4}{|l|}{ Response } \\
\hline CR & $34(44)$ & $84(36)$ & \\
\hline PR & $22(29)$ & $70(30)$ & .58 \\
\hline Failure (F) & 7 (9) & $23(10)$ & \\
\hline Not assessable (NA) & $14(18)$ & $56(24)$ & \\
\hline \multicolumn{4}{|l|}{ Response } \\
\hline $\mathrm{CR} / \mathrm{PR}$ & $56(73)$ & $154(66)$ & .326 \\
\hline F/NA & $21(27)$ & $79(34)$ & \\
\hline
\end{tabular}


A

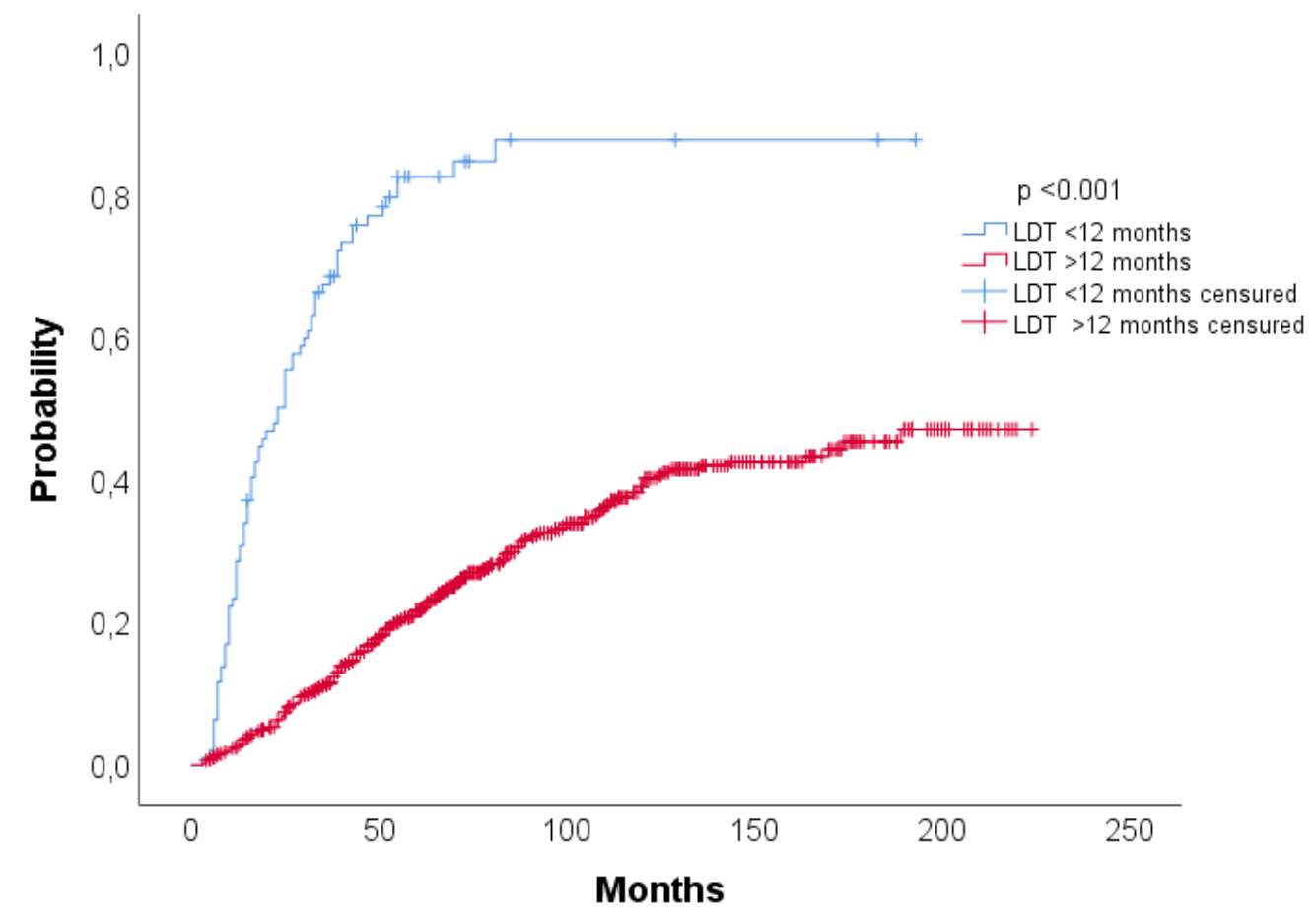

B

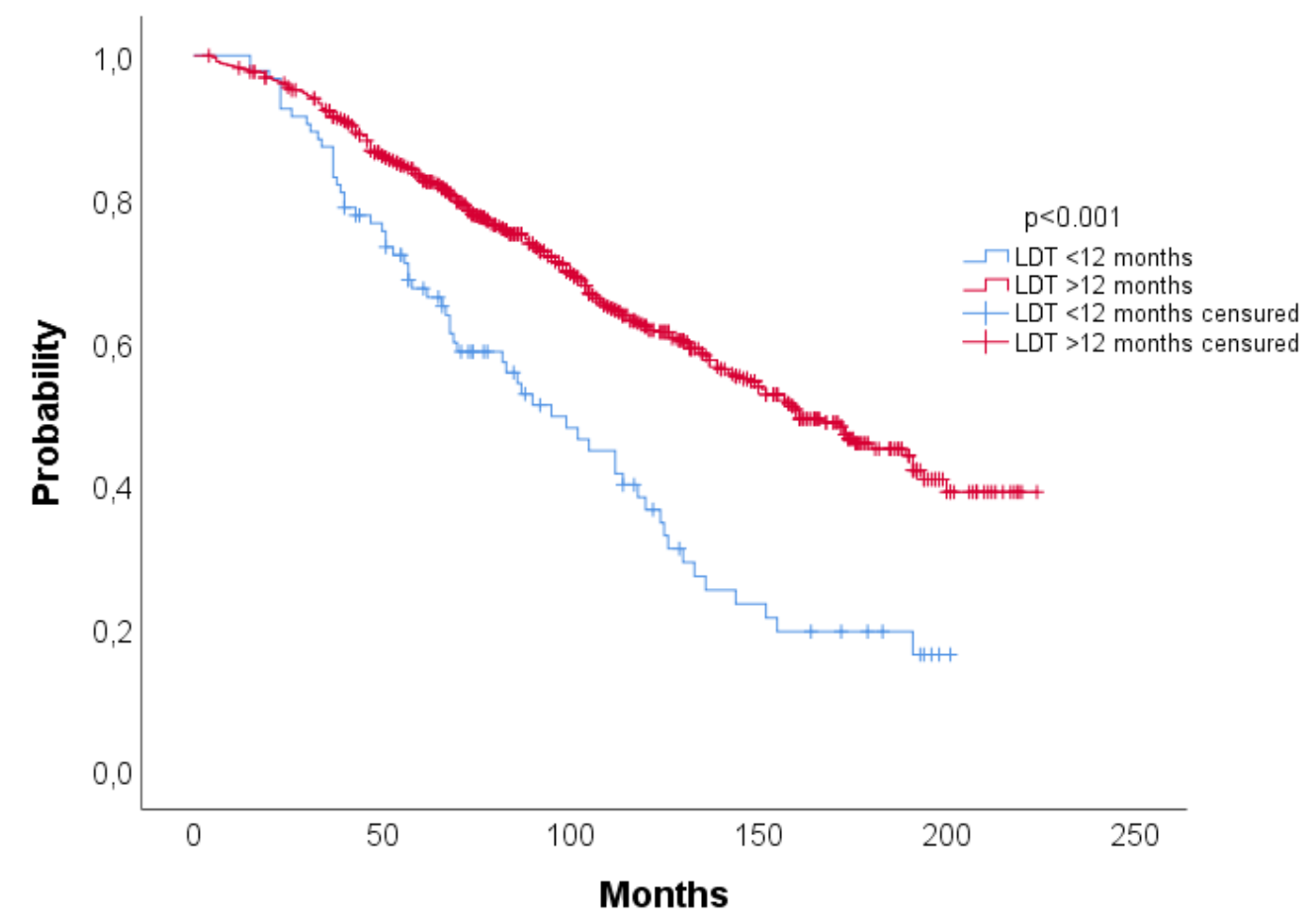


Table 2.

Univariate análisis and multivariate regression for time-to-first-therapy (TTFT) and overall survival (OS) according to clinico-biological features in 848 patients with CLL.

\begin{tabular}{|c|c|c|c|c|c|c|c|}
\hline \multirow[b]{3}{*}{ Parameter } & \multirow[b]{3}{*}{ Risk category } & \multicolumn{3}{|c|}{ Time-to-first-therapy (TTFT) } & \multicolumn{3}{|c|}{ Overall survival (OS) } \\
\hline & & \multirow{2}{*}{$\begin{array}{c}\begin{array}{c}\text { Univariate } \\
\text { Log-rank }\end{array} \\
P \text { value }\end{array}$} & \multicolumn{2}{|c|}{$\begin{array}{l}\text { Multivariate } \\
\text { Cox regression }\end{array}$} & \multirow{2}{*}{$\begin{array}{c}\begin{array}{c}\text { Univariate } \\
\text { Log-rank }\end{array} \\
P \text { value }\end{array}$} & \multicolumn{2}{|c|}{$\begin{array}{c}\text { Multivariate } \\
\text { Cox regression }\end{array}$} \\
\hline & & & $\begin{array}{c}\mathrm{HR} \\
(95 \% \mathrm{Cl})\end{array}$ & $P$ value & & $\begin{array}{c}\mathrm{HR} \\
(95 \% \mathrm{Cl})\end{array}$ & $P$ value \\
\hline Age & $>65$ years & NS (0.67) & - & NS (0.09) & $<.001$ & $2.9(2.1-3.9)$ & $<0.001$ \\
\hline Rai stage & I-IV & $<.001$ & $2.6(2.0-3.5)$ & $<.001$ & $<.001$ & - & NS $(0.34)$ \\
\hline $\mathrm{B} 2 \mathrm{M}$ & $>U N L$ & $<.001$ & $1.5(1.1-2.1)$ & .005 & $<.001$ & $2.1(1.5-2.8)$ & $<.001$ \\
\hline LDT & $\leq 12$ months & $<.001$ & $4.3(3.0-6.1)$ & $<.001$ & $<.001$ & $1.5(1.1-2.1)$ & .017 \\
\hline IGHV & Unmutated & $<.001$ & $3.0(2.3-4.1)$ & $<.001$ & $<.001$ & $1.8(1.3-2.3)$ & $<.001$ \\
\hline FISH & del11q or del17p & $<.001$ & - & NS (0.7) & $<.001$ & $1.9(1.3-2.8)$ & .002 \\
\hline TP53 & Mutated & .003 & - & NS (.14) & $<.001$ & $1.6(1.1-2.6)$ & .045 \\
\hline NOTCH1 & Mutated & $<.001$ & $1.6(1.1-2.4)$ & .012 & $<.001$ & - & NS $(0.27)$ \\
\hline SF3B1 & Mutated & $<.001$ & $1.99(1.2-2.9)$ & .008 & 0.25 & . & NS \\
\hline ATM & Mutated & $<.001$ & - & NS (.051) & 0.35 & - & NS \\
\hline
\end{tabular}


